\title{
Fractional revivals of the quantum state in a tight-binding chain
}

\author{
Bing Chen ${ }^{1}$, Z. Song ${ }^{1, a}$ and C.P. Sun ${ }^{1,2, a, b}$ \\ ${ }^{1}$ Department of Physics, Nankai University, Tianjin 300071, China and \\ 2 Institute of Theoretical Physics, Chinese Academy of Sciences, Beijing, 100080, China
}

\begin{abstract}
We investigate the time evolution of a Gaussian wave packet (GWP) in a tight-binding chain with a uniform nearest neighbor (NN) hopping integral. Analytical investigations and numerical simulations show that fractional revivals of the quantum state occur in this system, i.e., at appropriate times, a GWP can evolve into many copies of the initial state at different positions. The application of this quantum phenomenon to quantum information transfer in solid-state systems is discussed.
\end{abstract}

PACS numbers: 03.65.Ud, 03.67.MN, 71.10.FD

\section{INTRODUCTION}

Fractional revivals of a quantum state occur when the wave function evolves in time to a state, at a specific instant between two full revivals, which can be described by a superposition of states with equal amplitudes, each of which has the same shape as that of the initial wave packet, yet with different spatial distributions. The fractional revival is an interesting phenomenon in quantum mechanics, which has no analog in classical physics. It has been studied extensively, both theoretically [1, 2, 3, 4, 5] and experimentally [6, 7, 8, 9]. Most of the studies have been devoted to continuous systems, such as the Coulomb potential and the infinite square well. In this paper, we will focus on the phenomenon of fractional revival in a discrete solid-state system. As an illustration, a simple tight-binding chain with a uniform nearest neighbor (NN) hopping integral is investigated analytically and numerically. Such a model is used to describe the Bloch electronic system in condensed matter physics and now the qubit array relevant to quantum information applications due to its equivalence with the $X Y$ spin chain. It is found that fractional revivals of a Gaussian wave packet (GWP) occur in a discrete system. This opens up the possibility of performing high-fidelity quantum information transfer (QIT) and creating longrange entanglement by employing this novel feature of the time evolution in this solid-state system.

Quantum information processing in solid-state systems has attracted widespread attention because of the potential scalability of devices. Within this context, quantum state transfer (QST) from one place to another in such a system becomes a crucial issue and has been analyzed theoretically [10, 11, 12, 13, 14, 16, 17, 18, 19, 20, 21, 22]. A great advantage of this approach is that no dynamical controls are needed after one prepares the quantum state. In one of the pioneering works [10], Bose considered a regular one-dimensional spin chain with Heisenberg interactions, which is able to transfer a quantum state over a reasonable distance with the aid of a distillation process. Since then, a number of interesting proposals have been made for quantum communication through spin systems to improve the fidelity of the QST. One of them is to choose the proper modulation of the coupling strengths as suggested in [16, 17, 18]. In such a system, although an arbitrary local quantum state will spread as the time evolves, after a period of time the dispersed amplitudes will "refocus" at the receiving location of the chain. So perfect state transfer can be realized. Another approach makes use of gapped systems. The advantage of these schemes is that the intermediate spins are only virtually excited. In this case, the two separated qubits are coupled and realize the entanglement of two points [11]. This ensures that the transfer of the single-qubit state is achieved with a very high fidelity.

It should be pointed that QST and QIT are two different concepts. QST means a local quantum state changes its location. It usually corresponds to the translation or reflection of the initial wave function. On the other hand, typical quantum information means the way (or mode) of the superposition of two orthogonal states. A complete QST can achieve perfect QIT. However, it is sufficient but not necessary.

So far, almost all the proposed schemes for QIT are based on the fact that a properly designed qubit array can provide a unitary evolution operator $U(t)$ which can accomplish the task of transferring the local state $\left|\psi_{A}\right\rangle$ at position $A$ to the target state $\left|\psi_{B}\right\rangle$ located at $B$ via the process of time evolution $\left|\psi_{B}\right\rangle=U(t)\left|\psi_{A}\right\rangle$.

The phenomenon of quantum revival is an example of this process, which transmits the complete local state over the distance if $\left\langle\psi_{B}|U(t)| \psi_{A}\right\rangle=1$ (see Fig. 1a). In this case, we can say that perfect QST and QIT are both achieved. However, theoretically, if the data bus guarantees the partial revival, i.e., $U(t)\left|\psi_{A}\right\rangle=u_{B}\left|\psi_{B}\right\rangle+$ $u_{C}\left|\psi_{C}\right\rangle$ with $\left\langle\psi_{B} \mid \psi_{C}\right\rangle=0$ (see Fig. 1b), the quantum information encoded in the target state $\left|\psi_{B}\right\rangle$ can be also extracted from the final state $U(t)\left|\psi_{A}\right\rangle$. In this case, the quantum state can not be transferred perfectly, but perfect QIT is accomplished. We will show that such a scheme can be realized based on the fractional revival of a quantum state.

In general, there are two ways to employ the tightbinding model as a data bus for the QIT: (1) The qubit array is usually described by a Heisenberg spin chain system [10]. Within the context of quantum state transfer, only the dynamics of a single magnon are relevant. Thus in the single-magnon invariant subspace, this model can 


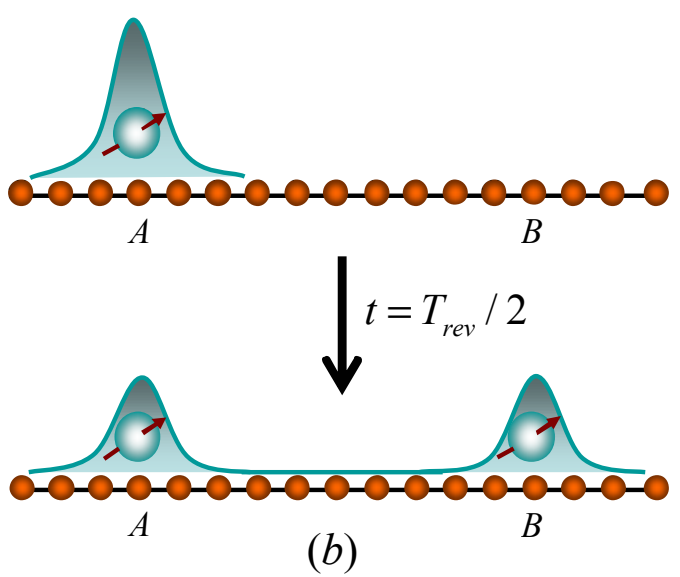

FIG. 1: (Color online) Schematic illustrations of quantum information transfer implemented by the revivals of a polarized electronic wave packet: (a) at instant $t=T_{\text {rev }}$, the initial wave packet at $A$ is in full revival at $B$; (b) at instant $t=T_{\text {rev }} / 2$, it is in half revival at $B$.

be mapped into the single spinless fermion tight-binding model. The quantum information is encoded in the superposition of the single and zero particle states. (2) On the other hand, the quantum information can also be encoded in the polarization of the Bloch electron (Fig. 1). If the spin state of the Bloch electron is a conserved quantity for the Hamiltonian of the medium, the spin state cannot be influenced during the propagation, no matter how the spatial shape of the wave function changes 23 . In this case, the locality of the final state is crucial. We will discuss the two schemes in detail in section IV based on the formalism of the fractional revival in the tightbinding model.

This paper is organized as follows. In Sec. II the model is presented. In Sec. III we formulate the general theory for the fractional revivals of a GWP in the tight-binding model. In Sec. IV we show numerical results that substantiate the analytical results. Sec. V is devoted to the application of the theory for QST and QIT. Sec. VI is the summary and discussion.

\section{MODEL HAMILTONIAN}

For completeness, we consider a non-interacting spin$1 / 2$ fermion system on a one-dimensional lattice, which is a simple tight-binding $N$-site chain with uniform $\mathrm{NN}$ hopping integral $-J$. The Hamiltonian can be written as

$$
H_{e}=-J \sum_{j=1 ; \sigma=\uparrow, \downarrow}^{N-1}\left(c_{j, \sigma}^{\dagger} c_{j+1, \sigma}+\text { h.c. }\right),
$$

where $c_{j, \sigma}^{\dagger}$ denotes the fermion creation operator at $j$-th site with spin $\sigma=\uparrow, \downarrow$. Because this Hamiltonian does not contain a spin-spin interaction, the polarization of the spin of an electron is not changed as time evolves. Then the problem about the transfer of the spin state can be reduced to the issue of charge transfer. In this sense, we can concentrate on the spinless fermion model

$$
H=-J \sum_{j=1}^{N-1}\left(a_{j}^{\dagger} a_{j+1}+\text { h.c. }\right),
$$

where $a_{j}^{\dagger}$ denotes the spinless fermion creation operator at $j$-th site, and the open boundary condition is applied. In section $\mathrm{V}$, the spin degree of freedom will be reconsidered for the discussion of QIT. Introducing the Fouriertransformed fermion operator

$$
\tilde{a}_{k}^{\dagger}=\sqrt{\frac{2}{N+1}} \sum_{j=1}^{N} \sin (k j) a_{j}^{\dagger},
$$

where $k=n \pi /(N+1), n=1,2, \ldots, N$, the Hamiltonian (2) can be diagonalized as

$$
\begin{aligned}
H & =\sum_{k} \epsilon_{k} \widetilde{a}_{k}^{\dagger} \widetilde{a}_{k}, \\
\epsilon_{k} & =-2 J \cos k,
\end{aligned}
$$

with the single-particle eigenstate

$$
|\widetilde{k}\rangle=\sqrt{2 /(N+1)} \sum_{j=1}^{N} \sin (k j)|j\rangle,
$$

where $|\widetilde{k}\rangle=\widetilde{a}_{k}^{\dagger}|0\rangle,|j\rangle=a_{j}^{\dagger}|0\rangle$.

In the large- $N$ limit, a discrete coordinate system approaches a continuous one. Correspondingly, state (5) is a standing wave which is the eigen wave function of the infinite square well. What is more, in lower energy region $k \sim 0$, the spectrum is $\epsilon_{k} \approx-2 J\left(1-k^{2}\right) \sim 2 J k^{2}$, which is very close to that of the infinite square well. This indicates that for low energies, the physics of the tight-binding chain is approximately the same as that of the infinite square well. Accordingly, for an initial state which can be expanded by the lower eigenstates, its time evolution should be similar to that of the infinite square well approximately, which is the typical paradigm to illustrate fractional revivals [3]. It implies that, for a wave packet with low energy, the well-defined fractional revival formalism should be valid in such a discrete coordinate system. 


\section{FRACTIONAL REVIVALS OF GWP}

In this section, we investigate the time evolution of a GWP in the tight-binding system analytically. Although the formalism presented in this paper has been well established for continuous systems, we derive it exactly to show that this formalism can be extended to discrete systems for some special states such as GWPs.

\section{A. General formalism}

We consider an initial state in the low-energy range, which can be expanded by the lower eigenstates. A typical state meeting this condition is the zero-momentum GWP, which can be expressed as

$$
\left|\psi\left(N_{0}\right)\right\rangle=\frac{1}{\sqrt{\Omega_{1}}} \sum_{j=1}^{N} e^{-\alpha^{2}\left(j-N_{0}\right)^{2} / 2}|j\rangle,
$$

where $\Omega_{1}=\sum_{j=1}^{N} e^{-\alpha^{2}\left(j-N_{0}\right)^{2}}$ is the normalization factor; $N_{0}$ denotes the center of the GWP and $1+\Delta / 2<$ $N_{0}<N-\Delta / 2$ ensures that the entirety of the GWP is situated within the chain approximately. The factor $\alpha$ determines the half-width

$$
\Delta=\frac{2 \sqrt{\ln 2}}{\alpha}
$$

of the GWP in real space and also the range of the spectrum related to its eigenstate expansion.

Firstly, we study the revival of a GWP in the framework of the spectrum-parity matching condition (SPMC) [22, 24], which is the basis for the investigation of the fractional revival. Actually, using the Fourier transformation (3), the GWP (6) can be written as

$$
\left|\psi\left(N_{0}\right)\right\rangle=\frac{1}{\sqrt{\Omega_{2}}} \sum_{k} \sin \left(k N_{0}\right) e^{-k^{2} / 2 \alpha^{2}}|\widetilde{k}\rangle,
$$

where $\Omega_{2}=\sum_{k} \sin ^{2}\left(k N_{0}\right) e^{-k^{2} / \alpha^{2}}$ is the normalization factor. For a system which has mirror symmetry, we have $[P, H]=0$, where $P$ is the reflection operator defined as $P|j\rangle=|N+1-j\rangle$. For the model Hamiltonian (2), which has the mirror symmetry, the eigenstate $|\widetilde{k}\rangle$ satisfies $P|\widetilde{k}\rangle=p_{k}|\widetilde{k}\rangle$ with $p_{k}= \pm 1$. So the mirror counterpart of the GWP (6) has the form

$$
\begin{aligned}
P\left|\psi\left(N_{0}\right)\right\rangle & =\left|\psi\left(N+1-N_{0}\right)\right\rangle \\
& =\frac{1}{\sqrt{\Omega_{2}}} \sum_{n}(-1)^{n+1} \sin \left(k N_{0}\right) e^{-k^{2} / 2 \alpha^{2}}|\widetilde{k}\rangle,
\end{aligned}
$$

with the relation $k=n \pi /(N+1)$. For the GWP (6) with a large enough $\alpha$, it can be expanded by the eigenstate $|\widetilde{k}\rangle$ with the eigenvalue $\varepsilon_{k}$ and parities $p_{k}$ in the following way:

$$
\varepsilon_{k}=n^{2} \Delta E, p_{k}=(-1)^{n^{2}},
$$

where $\Delta E=2 J \pi^{2} /(N+1)^{2}$ is the greatest common divisor of all the possible level differences. Obviously, the dispersion relation and the corresponding parity (10) satisfy the SPMC, which leads to the following conclusion: for an initial state $\left|\phi\left(N_{0}, 0\right)\right\rangle=\left|\psi\left(N_{0}\right)\right\rangle$, at the instant $t=T_{\text {rev }}=\pi / \Delta E$, it evolves into

$$
\left|\phi\left(N_{0}, \frac{\pi}{\Delta E}\right)\right\rangle=P\left|\phi\left(N_{0}, 0\right)\right\rangle .
$$

Here we define $T_{\text {rev }}$ as the revival time, at which the wave packet revives as the mirror reflection of the initial one. This is the same as in an infinite square well, and is usually called a full revival.

In the following, we will show that fractional revival of the GWP also occurs in the tight-binding model. We start our investigation with the time evolution of the GWP $\left|\psi\left(N_{0}\right)\right\rangle$ in the system (2). At the time $t$, the initial state $\left|\phi\left(N_{0}, 0\right)\right\rangle$ has evolved into

$$
\begin{aligned}
\left|\phi\left(N_{0}, t\right)\right\rangle & =e^{-i H t}\left|\phi\left(N_{0}, 0\right)\right\rangle \\
& \simeq \frac{e^{i 2 J t}}{\sqrt{\Omega_{2}}} \sum_{k} \sin \left(k N_{0}\right) e^{-k^{2} / 2 \alpha^{2}-i 2 J k^{2} t}|\widetilde{k}\rangle .
\end{aligned}
$$

Here, we take $H \simeq-2 J \sum_{k}\left(1-k^{2}\right) \widetilde{a}_{k}^{\dagger} \widetilde{a}_{k}$ as the small$k$ approximation for GWP. Neglecting the overall phase $e^{i 2 J t}$, we have

$$
\left|\phi\left(N_{0}, t\right)\right\rangle \simeq \frac{1}{\sqrt{\Omega_{2}}} \sum_{k} \sin \left(k N_{0}\right) e^{-k^{2} / 2 \alpha^{2}-i 2 J k^{2} t}|\widetilde{k}\rangle .
$$

The shape of $\left|\phi\left(N_{0}, t\right)\right\rangle$ in real space as the result of the interference of the standing waves $|\widetilde{k}\rangle$ depends on the time $t$ and $N_{0}$. In general, the feature of revival is characterized by the autocorrelation function $A(t)=$ $\left\langle\phi\left(N_{0}, 0\right) \mid \phi\left(N_{0}, t\right)\right\rangle$. In this paper, we choose another quantity, the mirror fidelity

$$
F(t)=\left\langle\phi\left(N_{p 0}, 0\right) \mid \phi\left(N_{0}, t\right)\right\rangle
$$

where $N_{p 0}=N+1-N_{0}$ denotes the mirror position of $N_{0}$, since the revival of the wave packet at different locations is desirable for the task of quantum state transfer and the generation of entanglement. The fidelity is a quantity to characterize the QST.

Now we focus on the special instants $\tau=p T_{\text {rev }} / q$, where $p, q$ are two mutually prime integers. In the framework of quantum information, $F(\tau)=1$ indicates perfect quantum state transmission. At the moment $\tau$, the fidelity of QST can be expressed explicitly

$$
|F(\tau)|=\left.\left|\sum_{n}(-1)^{n+1}\right| a_{n}\right|^{2} e^{-i p n^{2} \pi / q} \mid,
$$

where $a_{n}=\sqrt{1 / \Omega_{2}} \sin \left(k N_{0}\right) e^{-k^{2} / 2 \alpha^{2}}$ is the expansion coefficient, which is the starting point of our discussion. Obviously, when $p=q$, we have $\tau=T_{\text {rev }}$ and $F(\tau)=1$. This result is in agreement with the prediction from the 
SPMC, and can be employed to perform the perfect QIT. As discussed in the introduction, it may be possible to accomplish QIT in the case that the quantum state is not transferred completely.

In the following, we will demonstrate it in the framework of the well-defined formalism of fractional revivals. It is easy to see that $\exp \left(-i p n^{2} \pi / q\right)$ is a periodic function with period $l$, i.e.

$$
e^{-i p(n+l)^{2} \pi / q}=e^{-i p n^{2} \pi / q},
$$

where $l$ is determined by $q$

$$
l=\left\{\begin{array}{c}
2 q(\operatorname{odd} q) \\
q(\operatorname{even} q)
\end{array}\right.
$$

Performing the Fourier transformation, we have

$$
e^{-i p n^{2} \pi / q}=\sum_{r=0}^{l-1} b_{r} e^{-i 2 n \pi r / l}
$$

where

$$
b_{r}=\frac{1}{l} \sum_{n=0}^{l-1} e^{i\left(2 n \pi r / l-p n^{2} \pi / q\right)} .
$$

A straightforward calculation shows that $b_{r}$ satisfies the relation

$$
b_{r}=b_{l-r},(r=1,2, \ldots, l / 2-1)
$$

Then at the instant $\tau$, the state $\left|\phi\left(N_{0}, 0\right)\right\rangle$ evolves into

$$
\begin{aligned}
\left|\phi\left(N_{0}, \tau\right)\right\rangle= & \frac{1}{\sqrt{\Omega_{2}}} \sum_{n} a_{n} \sum_{r=0}^{l-1} b_{r} e^{-i 2 n \pi r / l}|k\rangle \\
= & b_{0}\left|\psi\left(N_{0}\right)\right\rangle-b_{l / 2} P\left|\psi\left(N_{0}\right)\right\rangle \\
& +\sum_{r=1}^{l / 2-1} b_{r}\left[\left|\psi\left(N_{r}^{+}\right)\right\rangle+\left|\psi\left(N_{r}^{-}\right)\right\rangle\right]
\end{aligned}
$$

where $N_{r}^{ \pm}=N_{0} \pm 2(N+1) r / l$. It is clear that, at the time $\tau=p T_{\text {rev }} / q$, the initial GWP at $N_{0}$ has evolved into $l$ sub-GWPs. Each of them closely reproduces the shape of the initial one but at the positions $N_{p 0}, N_{r}^{ \pm}$ $(r=0,1,2, \ldots, l / 2-1)$ and the probability of the subGWP at $N_{r}^{ \pm}\left(N_{p 0}\right)$ is $\left|b_{r}\right|^{2}\left(\left|b_{l / 2}\right|^{2}\right)$. Eq. (21) shows that the evolved state at time $\tau$ is the superposition of as many as $l$ "clones" of the initial wave packet with amplitude $b_{r}(r=0,1, \cdots, l / 2-1)$ and different spacial positions. The final state $\left|\phi\left(N_{0}, \tau\right)\right\rangle$ is formed in the following process. First, split the original wave packet into $l$ copies of the initial wave packet, each with the probability $\left|b_{r}\right|^{2}$. Then translate the copies to the positions $N_{s}^{ \pm}=N_{0} \pm 2(N+1) s / l,(s=0,1, \ldots, l / 2)$. For the sub-GWPs with $N_{s}^{ \pm}$beyond the chain, i.e., $N_{s}^{+}>N$ or $N_{s}^{-} \leq 0$, we have $\left|\psi\left(N_{s}^{+}\right)\right\rangle=e^{i \pi} P\left|\psi\left(N_{s}^{+}-N\right)\right\rangle$ and $\left|\psi\left(N_{s}^{-}\right)\right\rangle=e^{i \pi}\left|\psi\left(\left|N_{s}^{-}\right|\right)\right\rangle$from (8). This indicates that
$\left|\psi\left(N_{s}^{ \pm}\right)\right\rangle$will be reflected with $\pi$-shift when $N_{s}^{ \pm}$is beyond the chain, and there are always two sub-GWPs at the initial position $N_{0}$ and its mirror place $N_{p 0}$ respectively.

Defining the function

$$
f\left(N_{A}, N_{B}\right)=\left\langle\phi\left(N_{A}, 0\right) \mid \phi\left(N_{B}, 0\right)\right\rangle,
$$

thus the corresponding fidelity is

$$
\begin{aligned}
F(\tau) & =\left\langle\phi\left(N_{p 0}, 0\right) \mid \phi\left(N_{0}, \tau\right)\right\rangle \\
& =-b_{l / 2}+\sum_{r=1, \lambda= \pm}^{l / 2-1} b_{r} f\left(N_{p 0}, N_{r}^{\lambda}\right)
\end{aligned}
$$

Here, term $\left\langle\phi\left(N_{0}, 0\right) \mid \phi\left(N_{p 0}, 0\right)\right\rangle$ has been ignored since we are only interested in the case with $\left|N_{p 0}-N_{0}\right| \gg \Delta$. Furthermore, from the relation

$$
b_{r}=e^{-i 2 \pi(r / l+p / q)} b_{r^{\prime}}
$$

where $r^{\prime}=r+2 p l / q$, we have

$$
\left|b_{r}\right|^{2}=\frac{1}{q}
$$

i.e., the probability of each sub-packet is $1 / q$. However, Eq. (21) shows that the final $l$ sub-GWPs may be not orthogonal due to the reflection and then their superposition determines the shape of the final state. On the other hand, the overlap of two neighbor GWPs should affect the shape of the final state if the sub-GWPs are too numerous. We will discuss this in Sec. V with the aid of numerical simulation. Actually, the above conclusion is also valid for an arbitrary initial state which satisfies the low-energy condition.

\section{B. Application of the formalism}

In the following, our aim is to apply the formalism developed above to several special cases, which should be useful for the transmission of quantum information. From the above analysis, we have $|F(\tau)|=1$ at $\tau=T_{\text {rev }}$, which shows that the initial GWP can be revived completely at this instant. However, according to the formalism, $T_{\text {rev }}$ is not the shortest period to perform perfect state transfer. If a proper $N_{0}$ is chosen, a shorter period can be obtained. Here we investigate the case with $N_{0}=N / 3$ to illustrate this point. When we consider the case $p / q=1 / 3$, the corresponding period of the Fourier transformation in Eq. (17) is $l=6$. Then at time $\tau=T_{\text {rev }} / 3$, we have

$$
\left|\phi\left(\frac{N}{3}, \frac{T_{r e v}}{3}\right)\right\rangle=e^{-i \pi / 3} P\left|\psi\left(\frac{N}{3}\right)\right\rangle .
$$

It shows that at $\tau=T_{\text {rev }} / 3$ the initial GWP recurs totally at its mirror counterpart. 
This result can also be explained in the framework of the SPMC. Actually, for the eigenstate expansion of the initial GWP $|\psi(N / 3)\rangle$, it is easy to find that the expansion coefficients of the levels $n=3 m$ ( $m$ is the integer) vanish. Then the greatest common divisor of the effective levels for the state $|\psi(N / 3)\rangle$ is $\Delta E_{\text {eff }}=6 J \pi^{2} /(N+1)^{2}$ $=3 \Delta E$, and the corresponding recurrence period $T_{r e v}^{\prime}=$ $T_{\text {rev }} / 3$. A similar analysis can be applied to the case of the initial state $|\psi(N / 2)\rangle$. In this case, the expansion coefficients of the levels $n=2 m$ vanish, and the corresponding recurrence period $T_{r e v}^{\prime}=T_{\text {rev }} / 8$.

Except these special cases, the greatest common divisor for the effective levels of all GWPs at other positions remains $\Delta E$. Nevertheless, if the number of the levels, which determines the greatest common divisor to be $\Delta E$ is few, an "effective" greatest common divisor should govern the recurrence time dominantly. For example, if we take $N_{0}=N / m,(m>3)$, we also notice large partial revivals at $\tau=T_{\text {rev }} / \mathrm{m}$. For $N_{0}=N / 4$, at $\tau=T_{\text {rev }} / 4$ the wave packet evolved into

$$
\begin{aligned}
& \left|\phi\left(\frac{N}{4}, \frac{T_{\text {rev }}}{4}\right)\right\rangle \\
= & \left(b_{0}-b_{1}\right)\left|\psi\left(\frac{N}{4}\right)\right\rangle+\left(b_{1}-b_{2}\right) P\left|\psi\left(\frac{N}{4}\right)\right\rangle
\end{aligned}
$$

which represents four cloned small GWPs at positions $N / 4$ and $3 N / 4$. Note that the superposition leads to $\mid b_{0}-$ $\left.b_{1}\right|^{2}=(2-\sqrt{2}) / 4 \approx 0.146$ and $\left|b_{1}-b_{2}\right|^{2}=(2+\sqrt{2}) / 4$ $\approx 0.854$, which indicates that at the time $\tau=T_{\text {rev }} / 4$ the initial GWP splits into two sub-GWPs, and the one at $N_{p 0}$ is the large partial revival.

Another example to illustrate this mechanism is when the initial state is a superposition of two GWPs with $N_{0}=N_{A}, N_{B}$ respectively, i.e.

$$
\begin{aligned}
\left|\Phi\left(N_{A}, N_{B}\right)\right\rangle & =\frac{1}{\sqrt{2}}\left[\left|\psi\left(N_{A}\right)\right\rangle+\left|\psi\left(N_{B}\right)\right\rangle\right] \\
& =\sum_{k} \frac{e^{-k^{2} / 2 \alpha^{2}}}{\sqrt{2 \Omega_{2}}}\left[\sin \left(k N_{A}\right)+\sin \left(k N_{B}\right)\right]
\end{aligned}
$$

The levels with vanishing expansion coefficients are determined by

$$
\begin{aligned}
n\left(N_{A}+N_{B}\right) & =2 N k \\
\text { or } n\left(N_{B}-N_{A}\right) & =(2 k-1) N .
\end{aligned}
$$

One of the solutions is $N_{B}=2 N_{A}=2 N / 3$. The vanishing levels are $n=2 k$ and $n=3(2 k-1)$ with $k=1,2, \ldots$. Then the greatest common divisor is $24 \Delta E$. The wave packet will be revived at multiples of $T_{r e v} / 24$ for this case, as shown in Fig. 4b.

\section{NUMERICAL SIMULATIONS}

The analysis above is based on the assumption that the spectrum of the system is quadratic. However, this is only approximately true in the lower energy range. In order to demonstrate the fractional revival in the tightbinding system and to show how exact the approximation is, in this section we will exhibit numerical simulations for finite size systems.

We start our investigation from the revival and fractional revival in the general case. We consider the time evolution of an initial GWP with $\Delta=24$ and $N_{0}=50$ in the system with $N=500$. According to the formalism we have $T_{r e v}=(N+1)^{2} /(2 \pi J) \approx 4.0 \times 10^{4} / J$, which is taken as the unit of time $t$ in the numerical results. The fidelity $|F(t)|^{2}$ over the interval $t \in\left[0,6 T_{\text {rev }}\right]$ is plotted in Fig. 2 a. It shows that the fidelity has peaks around the instants $\tau=T_{r e v}, 3 T_{r e v}, 5 T_{r e v}, \ldots$, which is in agreement with the formalism in Sec. II. Interestingly, there exist many regular small peaks between two neighbor big peaks. We present the small peaks in Fig. $2 \mathrm{~b}$ to show the details of the small peaks. According to the general formalism, for local GWP, at instants $\tau / T_{\text {rev }}=1,1 / 2,1 / 3$, $1 / 4,1 / 5, \ldots$, the corresponding $|F(\tau)|^{2}$ should be equal to the values of $\tau / T_{\text {rev }}$. The plot in Fig. $2 \mathrm{~b}$ is in agreement with the analytical results with high accuracy. In order to demonstrate the mechanism of the fractional revival more explicitly, we calculate the profile of the evolved state

$$
\left|\phi_{i}\left(N_{0}, \tau\right)\right|=\left|\left\langle i \mid \phi\left(N_{0}, \tau\right)\right\rangle\right|
$$

at $\tau / T_{\text {rev }}$ as a function of the position $i$ and plot it in Fig. 3. From the analytical results, at instants $\tau / T_{\text {rev }}=1 / 5$, $1 / 4,1 / 3,1 / 2$, and 1 , the cloned sub-GWPs have the probabilities of $1 / 5,1 / 4,1 / 3,1 / 2$, and 1 , which result in the maximum values of the corresponding sub-GWPs to be $0.089,0.099,0.114,0.140$ and 0.198 . The numerical results, the number and the shapes of the sub-GWPs are in good accord with the theoretical prediction approximately. Now we turn our numerical investigation to the special cases. Let us look at a wave packet initially localized at $N_{0}=N / 3$. The result introduced in Eq. (26) shows that the initial GWP recurs totally at its mirror part at time $\tau=T_{\text {rev }} / 3$. The numerical result in Fig. 47 shows that the theoretical analysis is in agreement with the result of numerical simulation represented by $\left|F\left(T_{r e v} / 3\right)\right|^{2}=1$, which has also been well explained from the SPMC. On the other hand, we also demonstrate the evolution of $|\Phi(N / 3,2 N / 3)\rangle$ in Eq. (28) numerically. In Fig. $4 \mathrm{~b}$, it shows that the first revival time is around $T_{r e v} / 24$ which is in agreement with the analytical result. It also indicates that the proper choice of initial wave packet can revive in a shorter time, which can be used to transfer long-range entangled GWPs in the discrete system. Numerical simulation is also performed in the same system but with $N_{0}=N / 4$. The theoretical calculation shows that such an initial GWP should revive at $N_{p 0}=3 N / 4$ after the time $T_{r e v} / 4$ with a relatively higher fidelity 0.854 . The numerical results presented in Fig. $5 \mathrm{~b}$ and $\mathrm{b}$ depict the characteristics of the time evolution of the initial wave packet $|\psi(N / 4)\rangle$ via the fidelity $|F(t)|^{2}$ and the profile of the wave func- 

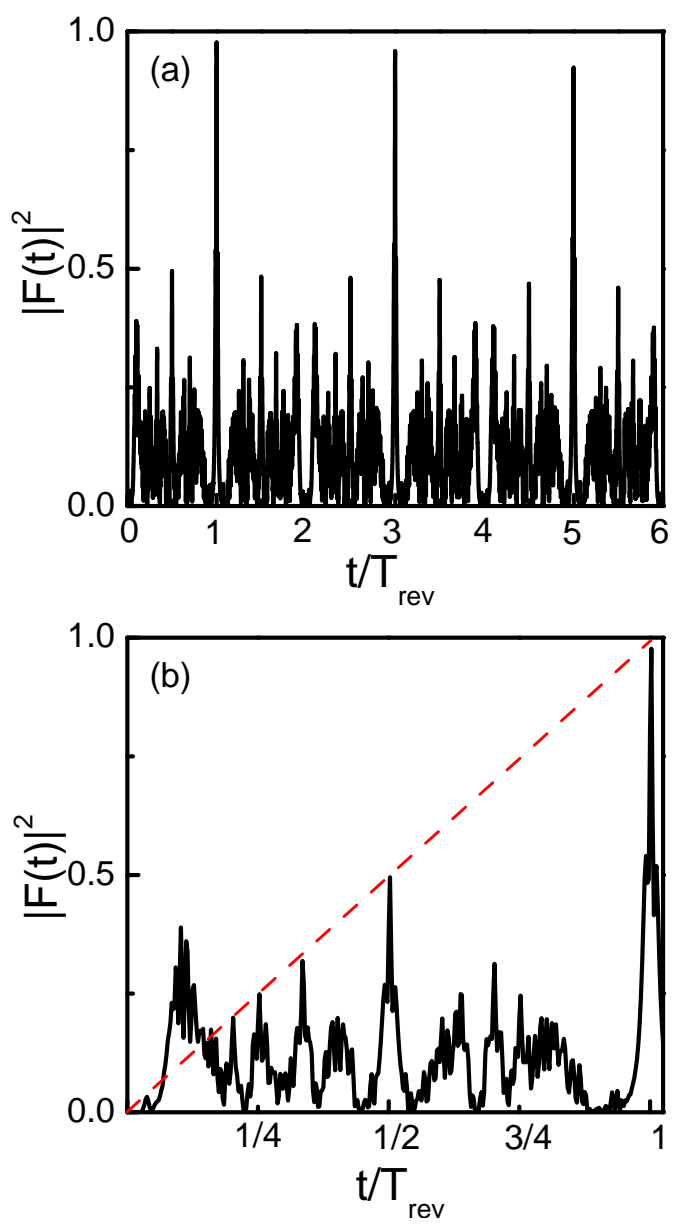

FIG. 2: (Color online) Plots of the square of fidelity, $|F(t)|^{2}$, for the initial GWP with $\Delta=24, N_{0}=50$ in the system with $N=500$. 2(b) is a part of 2(a) over one revival time. The dashed line indicates that the square of the fidelities at $\tau / T_{\text {rev }}=1 / 2,1 / 3,1 / 4, \ldots$ are approximately in a line.

tion $\left|\phi_{i}\left(N / 4, T_{r e v} / 4\right)\right|$. From the analytical result of Eq. (21), the behavior in Fig. 5 $\mathrm{a}$ and $\mathrm{b}$ can be explained. Actually, at the instant $T_{r e v} / 4$, the initial GWP splits into four sub-GWPs, with two of them being at $N_{p 0}$ and two at $N_{0}$. The final shape of the wave function should be two cloned initial wave packets with probabilities of 0.854 and 0.146 respectively. These will result in the two maxima of the wave functions, 0.183 and 0.076 around the positions $3 N / 4$ and $N / 4$.

Based on the numerical results presented in this section, we conclude that the fractional revival phenomena for local wave packets can be observed in the discrete system.

\section{FRACTIONAL FIDELITY OF QIT}

In general, there are two ways to employ the tightbinding model as a data bus for quantum information transfer: (1) The qubit array is usually described by a
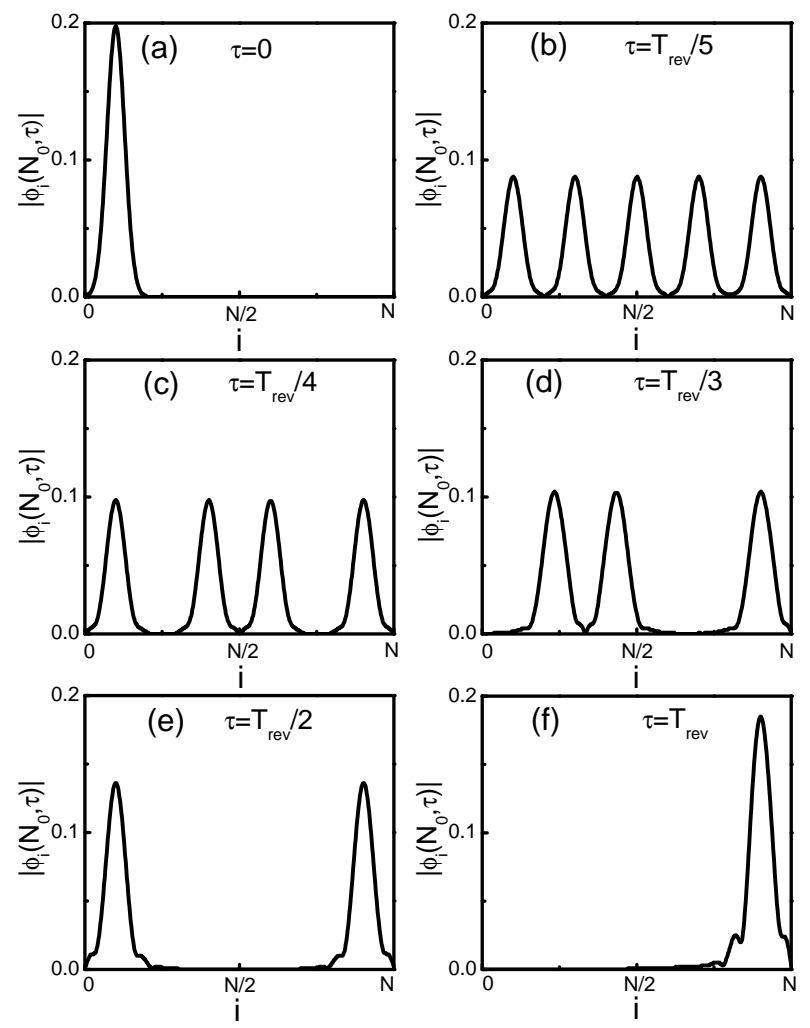

FIG. 3: The illustrations of the fractional revival via the time evolution of a GWP with $\Delta=24, N_{0}=50$ in the system with $N=500$. (a) $\tau=0$, (b) $\tau=T_{\text {rev }} / 5$, (c) $\tau=T_{\text {rev }} / 4$, (d) $\tau=T_{\text {rev }} / 3$, (e) $\tau=T_{\text {rev }} / 2$, (f) $\tau=T_{\text {rev }}$. It shows that at $\tau=T_{\text {rev }} p / q$, the GWP splits into several sub-GWPs at corresponding positions with the same shape as the initial one.

Heisenberg spin chain system [10]. Within the context of quantum state transfer, only the dynamics of the single magnon is relevant. Thus in the single magnon invariant subspace, this model can be mapped into a single spinless fermion tight-binding model. The quantum information is encoded in the superposition of the single and zero particle states. (2) On the other hand, the quantum information can also be encoded in the polarization of the Bloch electron (Fig. 11). If the spin state of the Bloch electron is a conserved quantity for the Hamiltonian of the medium, the spin state cannot be influenced during the propagation, no matter how the spatial shape of the wave function changes [23]. In this case, the locality of the final state is crucial. We will discuss the two schemes in detail in the following based on the formalism of the fractional revival in the tight-binding model.

\section{A. Scheme A: qubit array}

It is well known that, by employing the Jordan-Wigner transformation [15], the one-dimensional tight-binding chain with NN hopping is equivalent to a simple $X Y$ 

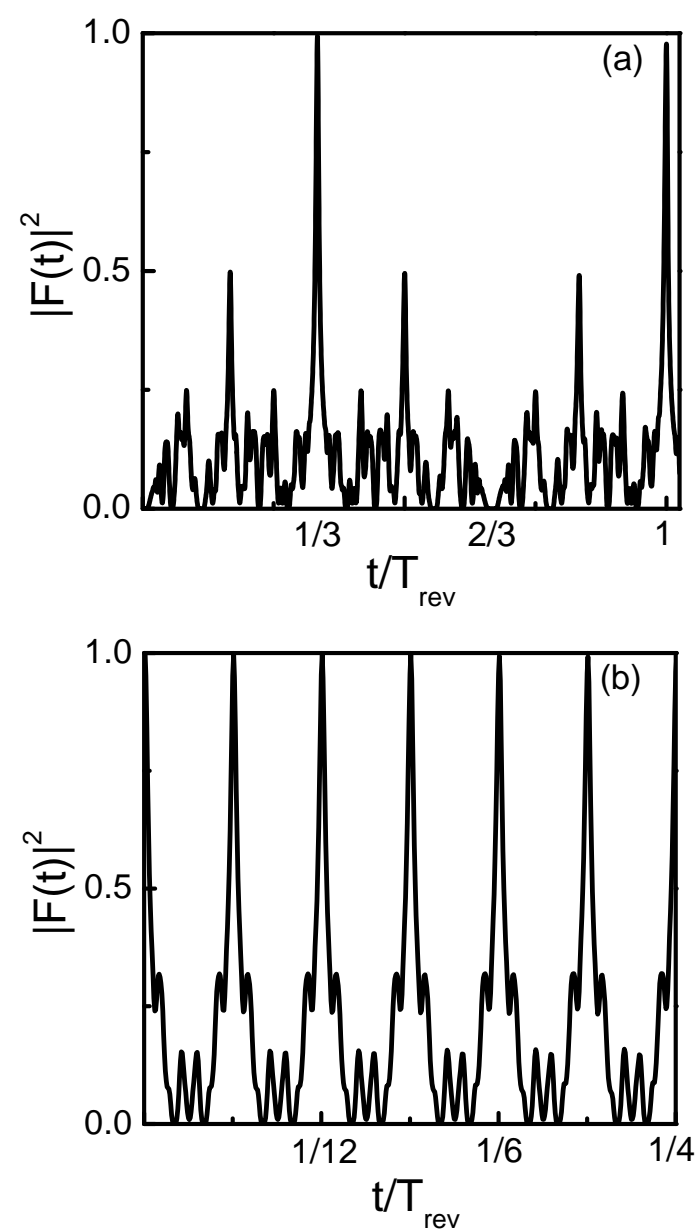

FIG. 4: (a) Plot of the square of fidelity $|F(t)|^{2}$, same as Fig. $2 \mathrm{~b}$, but for $N_{0}=N / 3$ over one revival time. (b) Plot of the square of fidelity over the time $\left[0, T_{r e v} / 4\right]$ for the initial state which is a superposition of two GWPs with $N_{0}=N / 3,2 N / 3$ respectively. It shows that for such an initial state, the revival period is reduced.

chain. Such a system is usually used to depict the physics of the qubit array. In this paper, the basis of the $X Y$ chain is in the form $\prod_{j}|\widetilde{n}\rangle_{j}$ with $\widetilde{n}=\widetilde{0}, \widetilde{1}$, i.e., $|\widetilde{1}\rangle_{j}=|\uparrow\rangle_{j}$ and $|\widetilde{0}\rangle_{j}=|\downarrow\rangle_{j}$. In general, the transmission of a qubit state from the location $A$ to $B$ can be regarded as the following process. The initial qubit state $\left|\psi_{A}\right\rangle=u|\tilde{1}\rangle_{A}+v|\tilde{0}\rangle_{A}$ is prepared at $A$. If one can find an operation $U_{A B}$ to realize

$$
U_{A B}\left|\psi_{A}\right\rangle=u|\tilde{1}\rangle_{B}+e^{i \varphi_{A B}} v|\widetilde{0}\rangle_{B},
$$

where $\varphi_{A B}$ is the known phase for a given system, we say that the qubit state is transferred from $A$ to $B$ perfectly. Then the perfect QIT is accomplished. Bose [10] proposed that the operation $U_{A B}$ can be achieved in the qubit array by the time evolution of the system based on the fact that the saturated ferromagnetic state $\prod_{j}|\widetilde{0}\rangle_{j}$
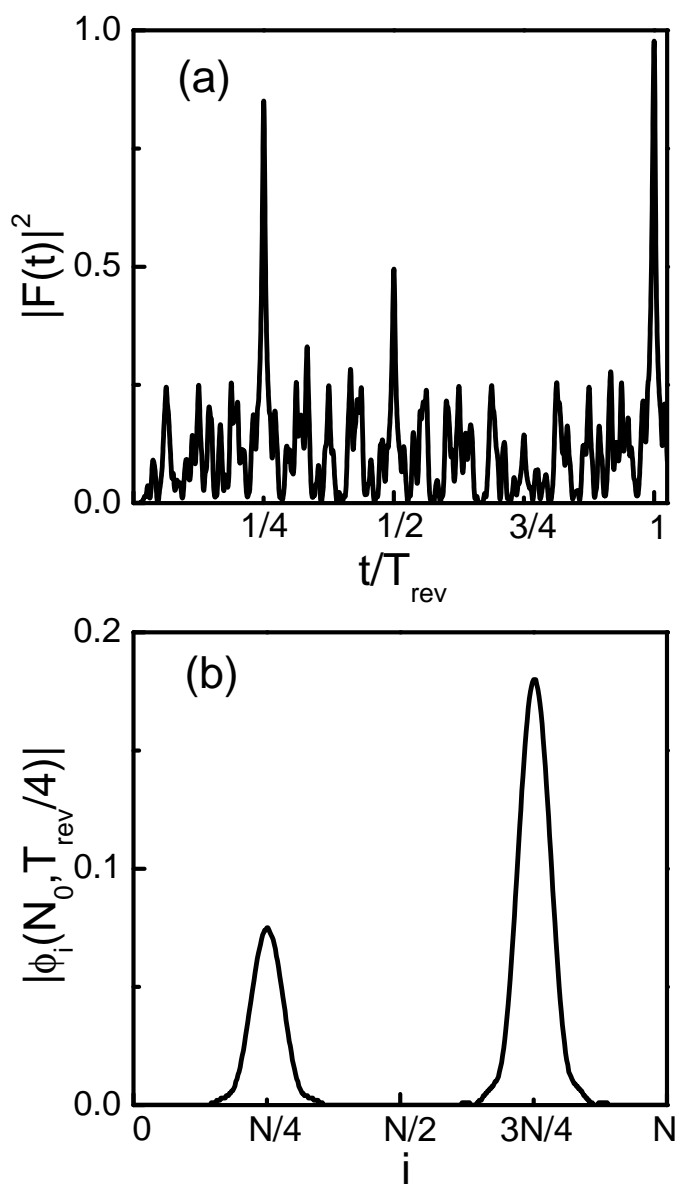

FIG. 5: (a) Plot of the square of fidelity $|F(t)|^{2}$, same as Fig. $2 \mathrm{~b}$, but for $N_{0}=N / 4$ over one revival time. (b) The profile of the evolved GWP at the time $\tau=T_{\text {rev }} / 4$.

is an eigenstate of the model. Furthermore, it is found that perfect state transfer can be implemented if the system meets the SPMC. Unfortunately, for an array to satisfy the SPMC, it requires modulation [16, 17, 18, 22] of the couplings between qubits, which is difficult to preengineer in experiments.

Nevertheless, we can consider the transferred state to be not a single-qubit state at a certain site but a superposition of single-qubit states localized in a small range of the coordinate space. Together with the saturated ferromagnetic state, the quantum information can be encoded in such a single magnon Gaussian wave packet at $N_{0}$

$$
\left|\psi_{N_{0}}\right\rangle=\frac{1}{\sqrt{\Omega_{1}}} \sum_{i} e^{-\alpha^{2}\left(i-N_{0}\right)^{2} / 2}\left(u|\widetilde{1}\rangle_{i}+v|\widetilde{0}\rangle_{i}\right) \prod_{j \neq i}|\widetilde{0}\rangle_{j} .
$$

This state contains the same quantum information as that of the single qubit state $\left|\psi_{A}\right\rangle$. So if the GWP (32) appears completely at another place, perfect QIT is accomplished. In the following, we will show that if the GWP (32) appears partially, perfect QIT can also be accomplished. 

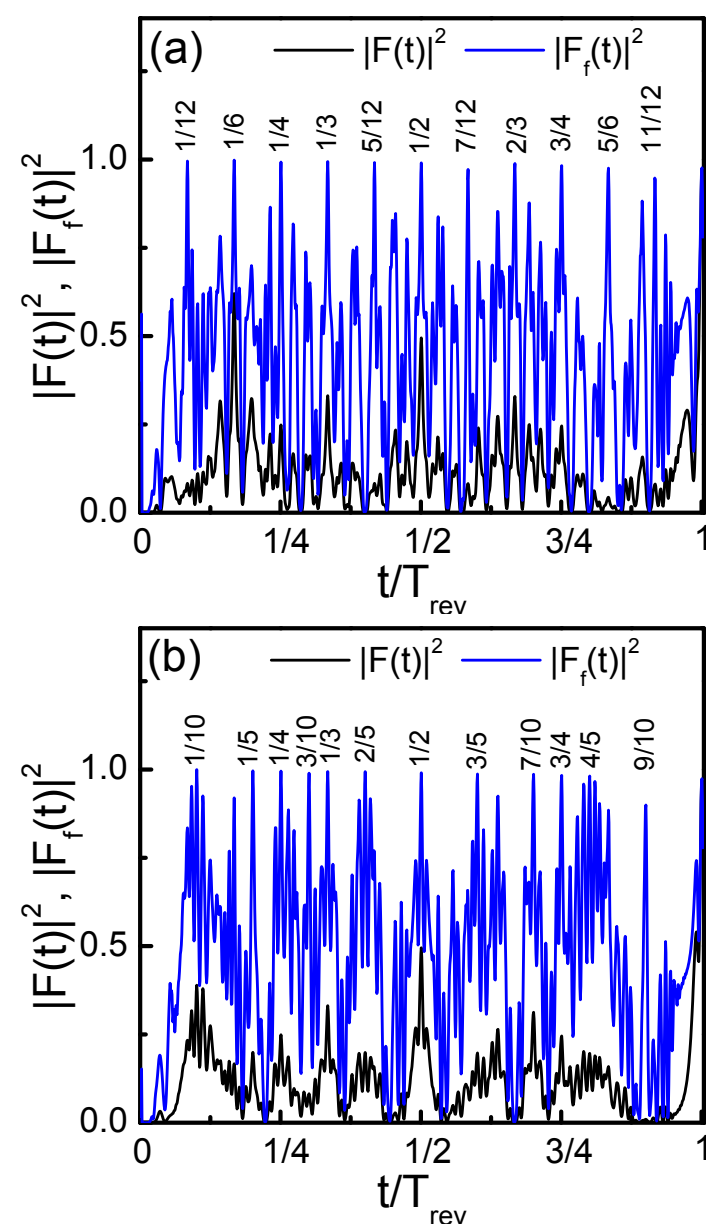

FIG. 6: (Color online) Plot of the square of the fractional fidelity $\left|F_{f}(t)\right|^{2}$ for the GWP over one revival time with $\Delta=$ 24 in the system with $N=500$ for (a) $N_{0}=N / 6$, (b) $N_{0}=$ $N / 10$. It shows that $\left|F_{f}(t)\right|^{2}$ is close to 1 at several specified instants.

According to the formalism of the fractional revival, we note that at $\tau=p T / q$ there always exists a cloned subGWP of the initial state at the mirror position, with the probability $\left|b_{l / 2}\right|^{2}$. From the point of view of quantum information, theoretically, the information of initial state encoded in the initial state by factors $u$ and $v$ has been transferred to its counterpart completely, although the fidelity of QST $F(\tau)$ may be far from 1.

In order to depict this fact, we introduce the fractional fidelity, which is expressed as

$$
\begin{aligned}
\left|F_{f}(\tau)\right| & =\frac{1}{\left|b_{l / 2}\right|}|F(\tau)| \\
& =\frac{1}{\left|b_{l / 2}\right|}\left|-b_{l / 2}+\sum_{r=1, \lambda= \pm}^{l / 2-1} b_{r} f\left(N_{p 0}, N_{r}^{\lambda}\right)\right|
\end{aligned}
$$

and is unity if the retrieved sub-GWP is the exact clone of the initial state. Then the QIT can be transferred perfectly, even the QST is not completely.

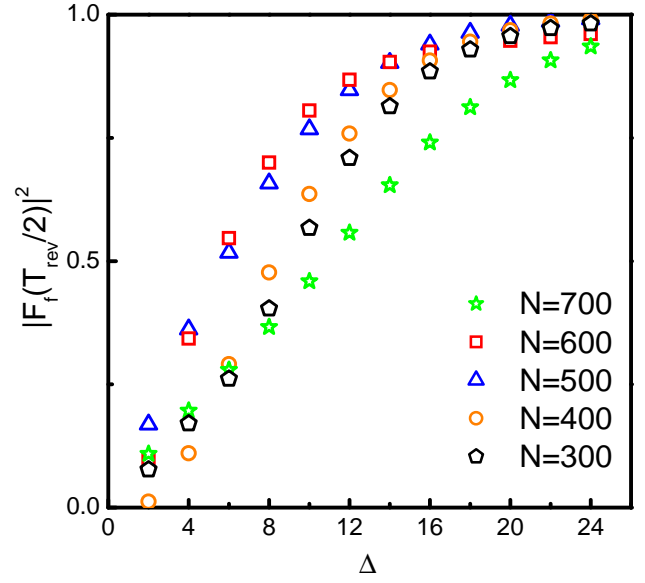

FIG. 7: (Color online) The square of fractional fidelity $\left|F_{f}\left(T_{\text {rev }} / 2\right)\right|^{2}$ versus $\Delta$ for systems with various lengths $N \in$ $[300,700]$. It shows that enlarging $\Delta$ can enhance the fractional fidelity.

To demonstrate the new definition of the fidelity and verify how much the initial state and the sub-GWP are alike, numerical simulation is performed in a finite system. The square of the fractional fidelity $\left|F_{f}(t)\right|^{2}$ for the propagation of a GWP with $\Delta=24$ from $N_{0}=N / 6$, $N / 10$ to $N_{p 0}=5 N / 6,9 N / 10$ in the system with $N=500$ within $\left[0, T_{r e v}\right]$ is plotted in Fig. 6. It is obvious that at many instants $\tau=T_{r e v} p / q$, the square of the fractional fidelity approximately equal to 1 , while $\left|F_{f}(\tau)\right|^{2}$ is still far from 1 for many possible $T_{\text {rev }} p / q$. We also notice that the initial position affects the results strongly. These phenomena can be explained as follows.

As discussed in Sec. III (A), the final state should be the superposition of the $l$ cloned sub-GWPs. Although there always exists a cloned sub-GWP at the position $N_{p 0}$, the overlap of these sub-GWPs affects $\left|F_{f}(\tau)\right|$. If the $l$ cloned sub-GWPs are well separated, i.e., $f\left(N_{p 0}, N_{r}^{ \pm}\right)=0$, then we have $\left|F_{f}(\tau)\right|=1$ directly from the Eq. (33). On the other hand, the orthogonality of the $l$ cloned sub-GWPs is determined by their number and positions, which depend on the factors $q$ and $N_{0}$. Obviously the smaller $l$ is, the more perfect the fractional revival is. However, in the case that the initial position $N_{0}$ and $l$ satisfy the condition

$$
\left(N+1-2 N_{0}\right) l=\text { integer } \times 2(N+1)
$$

the fractional revival should be still perfect. The Eq. (34) guarantees that the final state consists of several well separated cloned GWPs. This is in agreement with the numerical simulations. Fig. 6 a shows that $\left|F_{f}(\tau)\right|^{2} \sim 1$ at $\tau=T_{\text {rev }} p / 12(p=1,2, \cdots, 11)$, while it occurs at $\tau=T_{\text {rev }} p / 10(p=1,2, \cdots, 9)$ in Fig. 6b. 


\section{B. Scheme B: Flying qubit}

There is another scheme for QIT when the spin degree of freedom is considered. We can imagine an electronic wave packet with spin polarization as an analog of a photon "flying qubit", i.e. a polarized photon where the quantum information is encoded in its two polarization states. We define the solid-state "flying qubit", at the location $A$ in a quantum wire, as the superposition of two orthogonal Bloch electronic GWPs $|\uparrow\rangle_{A}$ and $|\downarrow\rangle_{A}$, where

$$
|\sigma=\uparrow, \downarrow\rangle_{A}=\frac{1}{\sqrt{\Omega_{1}}} \sum_{j} e^{-\alpha^{2}\left(j-N_{A}\right)^{2} / 2} c_{j, \sigma}^{\dagger}|0\rangle .
$$

Obviously, the two orthogonal states evolve independently. Then an arbitrary state

$$
|\Psi(0)\rangle_{A}=u|\uparrow\rangle_{A}+v|\downarrow\rangle_{A}
$$

will evolve to

$$
|\Psi(t)\rangle=u|\phi(\uparrow, t)\rangle+v|\phi(\downarrow, t)\rangle,
$$

where $|\phi(\sigma, t)\rangle=\sum_{j} f(j, t) c_{j, \sigma}^{\dagger}|0\rangle$ with $f(j, t)$ being a spin-independent function. The quantum information encoded in the spin state of the initial state (35) is carried along by the electron and unaffected by the transfer. Therefore, the initial state will be transferred to another location if $f(j, t)$ is known and is still a localized function. Of course, the simplest case is that $f(j, t)$ is a mirror or translation of the initial GWP [23]. In fact, if $f(j, t)$ is partially local at several places, the quantum information can be transported to multiple receivers. This fact indicates that such a system can be used for a "quantum fanout", which was recently proposed by A.D. Greentree, S.J. Devitt, and L.C.L. Hollenberg [25]. In our work, we only employ a simple open chain without any dynamic control. In this sense, it acts as a solid-state based splitter, entangler [26], and quantum fanout.

\section{Validity of the schemes}

In Sec. III, the analytical conclusion is only valid for lower energy GWPs. For an arbitrary GWP, the factor $\alpha$ determines the behavior of the final state as time evolves. As pointed above, for the GWP with narrow width at $k \sim$ 0 in momentum space, the effective dispersion relation is quadratic approximately. Then the width $\Delta$ of GWP in real space should affect the fractional fidelity of the fractional revival. Numerical simulation was employed to investigate the relationship between the fractional fidelity $F_{f}\left(\tau=T_{r e v} / 2\right)$ and $\Delta$ with the GWP transferring from
$N_{0}=50$ to $N_{0 p}=N-50$. The numerical results for $N=300,400,500,600$ and 700 are plotted in Fig. 7 . It shows that when $\Delta$ tends to $24, F_{f}\left(\tau=T_{\text {rev }} / 2\right) \sim 1$ for different sizes of system. Thus it indicates that when fractional revivals in the discrete system are employed for quantum information transmission, the width of the chosen GWP should be more than 24 times the lattice spacing.

\section{SUMMARY AND DISCUSSION}

In summary, we have studied the phenomenon of fractional revivals in a discrete system by theoretical analysis and numerical simulations of the evolution of a GWP in a tight-binding model. It is found that, for a proper chosen initial state, its fractional revival states have the same formalism as that in the infinite square well. On the other hand, numerical simulations show that the formulas of the theoretical analysis are very accurate for the GWP.

We also proposed the concept of the fractional fidelity $F_{f}(t)$ when the fractional revival phenomenon is exploited to achieve QST in the solid-state system. We showed that the fractional fidelity approximately equals to 1 at many instants. With an appropriately chosen width, the GWP with a polarized spin state can be regarded as a flying qubit in the solid-state system to implement quantum information transmission.

It is worthwhile to discuss the applicability of the scheme presented above. Experimentally, the tightbinding model can be realized by a quantum dot array, SQUID array, etc.. In a real system, quantum decoherence is the main obstacle to the experimental implementation of quantum information. In our scheme for quantum state transfer, the quantum decoherence time limits the scale of the quantum wire. For coupled quantum dots, experiments show that the coupling strength $J \sim 10$ meV [27]. Therefore, for a $N$-site chain, the revival pe$\operatorname{riod} T_{\text {rev }}=(N+1)^{2} /(J \pi) \approx 1.6 \times 10^{-11} N^{2} \mathrm{~ms}$. On the other hand, the decoherence time of a quantum dot is $\tau \sim 1 \mathrm{~ms}$ [28]. For example, considering a quantum dot array with $N=500$, we have $T_{\text {rev }} \approx 4 \times 10^{-6} \mathrm{~ms}$, which is much smaller than $\tau$. Moreover, if we perform $n$ times full revival within the decoherence time $\tau$, the maximal size is $2.5 \times 10^{5} / \sqrt{n}$. Therefore, for $n \sim 10^{4}$, the size of the system should be limited to $10^{3}$, which implies the applicability of the scheme in practice.

We acknowledge the support of the CNSF (grant No. 90203018, 10474104, 10447133), the Knowledge Innovation Program (KIP) of Chinese Academy of Sciences, the National Fundamental Research Program of China (No. 2001CB309310). [a] emails: songtc@nankai.edu.cn and suncp@itp.ac.cn [b] Internet www site: http://www.itp.ac.cn/ ${ }^{\sim}$ suncp 
[1] R.W. Robinett, Phys. Rep. 3921 (2004).

[2] I. Sh. Averbukh and N. F. Perelman, Phys. Lett. A 139, 449 (1989); Acta Phys. Pol. A 78, 33 (1990).

[3] David L. Aronstein and C.R. Stroud, Phys. Rev. A 55, 4526 (1997).

[4] G.S. Agarwal and J. Banerji, Phys. Rev. A 57, 3880 (1998).

[5] G.A. Vugalter, A.K. Das and V.A. Sorokin, Phys. Rev. A 66, 012104 (2002).

[6] O. Knospe and R. Schmidt, Phys. Rev. A 54, 1154 (1996).

[7] M.J.J. Vrakking, D.M. Villeneuve and A. Stolow, Phys. Rev. A 54, R37 (1996).

[8] Kevin F. Lee, D.M. Villeneuve, P.B. Corkum and E.A. Shapiro, Phys. Rev. Lett. 93, 233601 (2004).

[9] Michael Spanner, E.A. Shapiro, and Misha Ivanov, Phys. Rev. Lett. 92, 093001 (2004).

[10] S. Bose, Phys. Rev. lett. 91, 207901 (2003).

[11] Y. Li, T. Shi, B. Chen, Z. Song, C.P. Sun, Phys. Rev. A 71, 032309 (2005).

[12] Z. Song and C.P. Sun, Low Temperature Physics 31, 686 (2005).

[13] Alexandre G. M. Schmidt and M. G. E. da Luz, Phys. Rev. A 69, 052708 (2004).

[14] Peter Karbach and Joachim Stolze, Phys. Rev. A 72, 030301(R) (2005).

[15] P. Jordan and E. Wigner, Z. Phys. 47, 631 (1928); E. Lieb, T. Schultz, and D. Mattis, Ann. Phys. 16, 407 (1961); S. Katsura, Phys. Rev. 127, 1508 (1962); N. Nagaosa, Quantum Field Theory in Strongly Correlated Electronic Systems, (Springer-Verlag, Berlin Heidelberg,
Germany, 1999).

[16] M. Christandl, N. Datta, A. Ekert and A.J. Landahl, Phys. Rev. Lett. 92, 187902 (2004).

[17] M. Christandle, N. Datta T. C. Dorlas, A. Ekert, A. Kay and A.J. Landahl, Phys. Rev. A 71, 032312 (2005).

[18] C. Albanese, M.Christandle, N. Datta and A. Ekert, Phys. Rev. Lett. 93, 230502 (2004).

[19] Daniel Burgarth and Sougato Bose, New J. Phys. 7, 135 (2005).

[20] D. Burgarth and S. Bose, Physical Review A 71, 052315 (2005).

[21] T. Shi, B. Chen and Z. Song, Comm. Theor. Phys. 43, 795 (2005).

[22] T. Shi, Ying Li, Z. Song and C.P. Sun, Phys. Rev. A 71, 032309 (2005).

[23] S. Yang, Z. Song, and C.P. Sun, Phys. Rev. A 73, 022317 (2006).

[24] Y. Li, Z. Song, and C.P. Sun, quant-ph/0504175

[25] Andrew D. Greentree, Simon J. Devitt, and Lloyd C. L. Hollenberg, Phys. Rev. A 73, 032319 (2006).

[26] S. Yang, Z. Song, and C.P. Sun, quant-ph/062209.

[27] Guido Burkard and Daniel Loss, Phys. Rev. B 59, 2070 (1999); A.V.Onufriev and J.B.Marston, Phys. Rev. B 59, 12573 (1999); W Gvander Wiel, et al. New Journal of Physics 8, 28 (2006).

[28] Rogeriode Sousa and S.Das Sarma cond-mat/0211567, Daniel Loss and David P. DiVincenzo, Phy. Rev. A 57, 120 (1998); Hans-Andreas Engel, et al. Quantum Information Processing, Vol. 3, 115 (2004). 